\title{
CARACTERIZAÇÃO DE SUJEITOS QUE ACESSARAM UM HOSPITAL UNIVERSITÁRIO REGIONAL POR LESÃO ENCEFÁLICA ADQUIRIDA
}

\section{CHARACTERIZATION OF INDIVIDUALS WHO WENT TO A REGIONAL UNIVERSITY HOSPITAL DUE TO ACQUIRED BRAIN INJURY}

\section{Jodéli Pommerehn', Elenir Fedosse ${ }^{2}$}

\section{RESUMO}

O estudo descreve e relaciona perfil, fatores de risco e diagnóstico de 60 sujeitos acometidos por Lesões Encefálicas Adquiridas (LEA) que acessaram, no mês de março de 2015, um Hospital Regional do Rio Grande do Sul. É um estudo descritivo, exploratório e quantitativo, aprovado pelo Comitê de Ética em Pesquisa. A média de idade dos sujeitos foi 51,33 anos; maioria 53 (88,33\%) é da cor branca e $36(60 \%)$ do sexo masculino. O diagnóstico prevalente foi Acidente Vascular Cerebral hemorrágico 15 (25\%), 10 (16,7\%) por Coinfeccção Neurológica e 9 (15\%) Tumores de Sistema Nervoso Central. Tais episódios foram associados aos fatores de risco - hipertensão arterial sistêmica, diabetes mellitus, etilismo, tabagismo e Human Immunodeficiency Virus estiveram presentes em 45\%,20\%,20\%,20\%, 10\% respectivamente. Estes, decorrentes de causas evitáveis, o que significa que as ações preventivas relacionadas a doenças vasculares, infectocontagiosas e neoplásicas do Sistema Nervoso ainda não são eficientes.

Descritores: Lesões Encefálicas; Fatores de Risco; Assistência Hospitalar.

\begin{abstract}
This study to describe and relate profile, risk factors and diagnosis of 60 individuals affected by Acquired Brain Injuries (ABI) who accessed, in March 2015, a Regional Hospital, from of the State of Rio Grande do Sul. It is a descriptive, exploratory and quantitative study approved by the Research Ethics Committee. The average age of the individuals was 51.33 years; most of them, $53(88.33 \%)$ are white people and $36(60 \%)$ are male. The prevalent diagnosis was Hemorrhagic Stroke $15(25 \%)$, $10(16.7 \%)$ Coinfection Neurological and 9 (15\%) Central Nervous System Tumors. Such episodes were associated with risk factors - hypertension, diabetes mellitus, alcoholism, smoking and Human Immunodeficiency Virus were present in $45 \%, 20 \%, 20 \%, 20 \%, 10 \%$ respectively. These resulting from preventable causes, which means that preventive actions related to vascular, infectious and neoplastic Nervous System diseases are not yet effective.
\end{abstract}

Descriptors: Brain injury; Risk factors; Hospital Care.

${ }^{1}$ Mestre em Distúrbios da Comunicação Humana pela Universidade Federal de Santa Maria (UFSM), Santa Maria, RS, Brasil.

2 Doutora em Linguística pela Universidade Estadual de Campinas (UNICAMP), Campinas, SP, Brasil. 


\section{Introdução}

As transformações socioeconômicas advindas da revolução tecnológica e industrial modificaram o perfil de morbimortalidade da população mundial ${ }^{1}$. As mudanças de hábitos de vida e o envelhecimento populacional aumentaram a exposição aos fatores responsáveis pelo aparecimento de infecções ${ }^{2,3}$, Doenças Crônicas Não Transmissíveis (DCNT) ${ }^{4}$ - atualmente identificadas como condições crônicas de saúde e pelas agressões externas (acidentes de trânsito, quedas de altura, por exemplo) ${ }^{5,6}$ que podem provocar as chamadas - Lesões Encefálicas Adquiridas (LEA).

De acordo com Brain Injury Support ${ }^{7,8}$, a LEAé definida como uma lesão que ocorre no encéfalo após o nascimento e tais danos podem advir de Traumatismos Crânio-Encefálicos (TCE), Acidentes Vasculares Cerebrais (AVC), tumores, lesões medulares, síndromes, doenças degenerativas e infecções do Sistema Nervoso (SN) ${ }^{8}$.

As doenças cerebrovasculares foram responsáveis por 99.732 óbitos ocorridos, no Brasil, no ano de $2010^{9}$. Dentre essas doenças, o AVC vem se destacando como a segunda causa de morte, no Brasil e no mundo, com tendência de se manter nesta posição até 0 ano de $2030^{10}$.

As condições crônicas de saúde, importantes determinantes das doenças cardiovasculares, assim como as respiratórias, neoplasias e diabetes ${ }^{11}$ apresentam-se como grandes problemas de Saúde Pública global à medida que produzem altos índices de mortalidade ${ }^{11,12}$. Em 2008,63\% dos óbitos ocorridos, no mundo, foram relacionados às, então chamadas, DCNT ${ }^{13}$; essa proporção é mais elevada nos países de baixa e média renda ${ }^{11,12}$. No Brasil, por exemplo, foi evidenciado que, no ano de 2009 , as DCNT foram responsáveis por $72,4 \%$ dos óbitos registrados ${ }^{11}$.

As lesões por TCE também constituem um grave problema de Saúde Pública mundial; com uma incidência muito elevada na população adulta jovem, principalmente como resultado dos acidentes de viação/trânsito5,6. O TCE tem grande impacto na saúde da população em geral, tendo notória importância na morbidade e mortalidade pós-trauma, representando entre $15 \%$ e $20 \%$ das mortes em pessoas com idade entre 5 e 35 anos; é responsável por 1\% de todas as mortes em adultos ${ }^{14}$.

Outro desafio da saúde mundial é o contínuo crescimento da infecção da população por Human Immunodeficiency Virus (HIV), que pode desenvolver a AIDS (Síndrome da Imunodeficiência Adquirida) caracterizada pela diminuição quantitativa e qualitativa dos linfócitos T, fragilizando a atuação do sistema imunológico e aumentando a suscetibilidade a infecções por inúmeros microrganismos oportunistas, dentre elas, as coinfecções do Sistema Nervoso ${ }^{2,3}$.

As neoplasias do SN também apresentam importância epidemiológica devido à sua crescente incidência e elevada morbimortalidade ${ }^{15,16}$. Tais neoplasias representam 1,5\% de todos os cânceres e 2,4\% de todas as mortes por câncer ocorridas, anualmente, no mundo ${ }^{17}$.

Considerando as epidemiologias acima, estudos ${ }^{18,19}$ ressaltam a importância da realização de outros; é de extrema importância identificar idade, sexo e fatores de risco (entre eles: tabagismo, etilismo, uso de drogas ilícitas, obesidade, diabetes e hipertensão arterial sistêmica) associados aos acometimentos neurológicos ${ }^{18,19}$. Esse conhecimento é essencial para concentrar recursos técnicos e humanos em medidas de prevenção ${ }^{19}$ dos fatores de risco modificáveis e/ ou evitáveis e, assim, promover a qualidade de vida da população.

Além dos argumentos anteriormente usados, sabe-se que convém realizar estudos sobre LEA, pois, quando não resultam em óbitos, estão associados a morbidades como agravos cognitivos, motores e sensoriais que implicam mudanças no cotidiano e na qualidade de vida, as quais por sua vez exigem processo de reabilitação. Por isso, estudos com informações detalhadas acerca da etiologia, perfil dos sujeitos e acometimentos são importantes para gerar intervenções de acordo com as necessidades da população ${ }^{19,20}$.

Dessa forma, este estudo tem como objetivo descrever e relacionar o perfil, os fatores de risco e o diagnóstico neurológico de adultos acometidos por LEA que acessaram um Hospital Regional.

\section{Metodologia}

Esta pesquisa foi realizada em um Hospital Regional de Alta Complexidade do interior do Rio Grande do Sul (RS); foi desenvolvida após a aprovação, em 29 de janeiro de 2015, do Comitê de Ética em Pesquisa, da referida instituição, sob o número, 941.552, conforme a Resolução n 466/12 do Conselho Nacional de Saúde, referente à pesquisa com seres humanos. 
Este estudo é de caráter descritivo e exploratório com análise quantitativa; descreve e interpreta dados de identificação, fatores de risco e diagnóstico neurológico de 60 sujeitos internados por LEA.

A coleta de dados ocorreu no mês de março de 2015, tendo como critério de inclusão sujeitos com idade igual ou superior a 18 anos, de ambos os sexos, tendo como motivo de internação hospitalar uma LEA. Utilizou-se um formulário, especialmente elaborado para esta pesquisa, para levantamento das informações contidas nos prontuários, a saber: sexo, cor, idade, cidade de procedência, fatores de risco e diagnóstico. Ressalta-se que os diagnósticos foram confirmados por Ressonância Nuclear Magnética (RNM) e/ou Tomografia Computadorizada (TC) e laudos médicos.

O banco de dados foi elaborado no programa de computador "Excel" versão 2013 e os dados foram analisados pelo Software Statistica 9.1, por meio de análise descritiva.

\section{Resultados}

Durante o mês de março de 2015, 60 sujeitos (acometidos por LEA) foram internados no hospital pesquisado. A idade mínima foi de 18 anos e a máxima de 80; a média de idade foi de 51,33 anos (desvio padrão de 18,5). A maioria (75\%) tinha idade igual ou inferior a 69 anos, sendo que os demais (25\%) tinham idade igual ou inferior a 34 anos. Vide, na Tabela, 1 a relação entre faixa etária e LEA.

Tabela 1 - Faixa etária e LEA

\begin{tabular}{|c|c|c|c|c|c|c|c|}
\hline \multirow{2}{*}{$\begin{array}{c}\text { Variável } \\
\text { Faixa Etária }\end{array}$} & \multicolumn{7}{|c|}{ Lesão Encefálica Adquirida n(\%) } \\
\hline & AVCh & AVCi & $\begin{array}{c}\text { Lesão } \\
\text { neurológica por } \\
\text { coinfecção }\end{array}$ & $\begin{array}{l}\text { Tumores } \\
\text { de SNC }\end{array}$ & TCE & Outros & Total \\
\hline 18 a 30 & - & $1(20)$ & $5(50)$ & - & $3(37,5)$ & $4(30,7)$ & $13(22)$ \\
\hline 30 a 40 & - & - & $2(20)$ & $2(22,2)$ & $2(25)$ & - & $6(10)$ \\
\hline 40 a 50 & $4(26,6)$ & - & $3(30)$ & - & $2(25)$ & - & $9(15)$ \\
\hline 50 a 60 & $4(26,6)$ & - & - & $1(11,1)$ & - & $3(23,0)$ & $8(13)$ \\
\hline 60 a 70 & $5(33,3)$ & $2(40)$ & - & $2(22,2)$ & - & $6(46,1)$ & $15(25)$ \\
\hline 70 a 80 & $2(13,3)$ & $2(40)$ & - & $4(44,4)$ & $1(12,5)$ & - & $9(15)$ \\
\hline Total n (\%) & $15(25)$ & $5(8)$ & 10 (17) & $9(15)$ & 8 (13) & $13(22)$ & $60(100)$ \\
\hline
\end{tabular}

Legenda: AVCh (Acidente Vascular Cerebral hemorrágico); AVCi (Acidente Vascular isquêmico); SNC (Sistema Nervoso Central); TCE (Traumatismo Crânio Encefálico); Outras (mielites, fraturas vertebrais, lesões medulares, síndromes).

Quanto à cor, a maioria dos sujeitos 53 (88,33\%) era da cor branca; quanto ao sexo, 36 (60\%) eram do masculino. Confira, na Tabela 2, a relação entre LEA e sexo.

Tabela 2 - LEA e sexo

\begin{tabular}{c|c|c|c}
\hline Diagnóstico & $\begin{array}{c}\text { Sexo Feminino } \\
\mathbf{n ( \% )}\end{array}$ & $\begin{array}{c}\text { Sexo Masculino } \\
\mathbf{n ( \% )}\end{array}$ & Total n (\%) \\
\hline AVCh & $7(46,6)$ & $8(53,3)$ & $15(25)$ \\
\hline AVCi & $4(80)$ & $1(20)$ & $5(8,3)$ \\
\hline TCE & $2(25)$ & $6(75)$ & $8(13,3)$ \\
\hline Tumor de SNC & $2(22,2)$ & $7(77,7)$ & $9(15)$ \\
\hline Lesão neurológica por coinfecção & $4(40)$ & $6(60)$ & $10(16,7)$ \\
\hline Outros & $5(20,8)$ & $8(22,2)$ & $13(21,7)$ \\
\hline
\end{tabular}

Legenda: AVCh (Acidente Vascular Cerebral hemorrágico); AVCi (Acidente Vascular isquêmico); TCE (Traumatismo Crânio Encefálico); SNC (Sistema Nervoso Central); Outros (mielites, fraturas vertebrais, lesões medulares, síndromes). 
No que tange aos fatores de risco e idade obteve-se prevalência da HAS em adultos maduros (Tabela 3).

Tabela 3 - Fatores de risco à LEA relacionados à faixa etária

\begin{tabular}{|c|c|c|c|c|c|c|c|c|}
\hline Variável & \multicolumn{8}{|c|}{ Fatores de Risco n(\%) } \\
\hline Faixa Etária & $\begin{array}{c}\text { AVC no } \\
\text { passado }\end{array}$ & Dislipidemia & DM & $\begin{array}{l}\text { Drogas } \\
\text { ilícitas }\end{array}$ & Etilista & HAS & Tabagista & HIV \\
\hline 18 a 30 & $2(22,2)$ & - & $2(16,6)$ & $4(100)$ & $1(8,3)$ & $3(11,1)$ & $2(13,3)$ & $5(50)$ \\
\hline 30 a 40 & - & - & $1(8,3)$ & - & $1(8,3)$ & - & $1(6,6)$ & $2(20)$ \\
\hline 40 a 50 & - & $1(16,6)$ & - & - & $2(16,6)$ & $2(7,4)$ & $2(13,3)$ & $3(60)$ \\
\hline 50 a 60 & $4(44,4)$ & $2(33,3)$ & $4(33,3)$ & - & $2(16,6)$ & $8(29,6)$ & $2(13,3)$ & - \\
\hline 60 a 70 & $3(33,3)$ & $2(33,3)$ & $5(41,6)$ & - & $5(41,6)$ & $11(40,7)$ & $6(40)$ & - \\
\hline 70 a 80 & - & $1(16,6)$ & - & - & $1(8,3)$ & $3(11,1)$ & $2(13,3)$ & - \\
\hline Total n (\%) & $9(15)$ & $6(10)$ & $12(20)$ & $4(6,6)$ & $12(20)$ & $27(45)$ & $15(25)$ & $10(16,7)$ \\
\hline
\end{tabular}

Legenda: AVC (Acidente Vascular Cerebral); DM (Diabetes Mellitus); HAS (Hipertensão Arterial Sistêmica); HIV (Human Immunodeficiency Virus).

Quanto aos fatores de risco à LEA, identificou-se que o tabagismo e o etilismo foram importantes riscos no sexo masculino; para o sexo feminino foi a hipertensão arterial sistêmica (Vide Tabela 4). Ressalta-se que existiram sujeitos que apresentam mais de um fator de risco para a LEA.

Tabela 4 - Fatores de risco à LEA relacionados ao sexo

\begin{tabular}{c|c|c|c}
\hline Fatores de Risco & $\begin{array}{c}\text { Sexo Feminino } \\
\mathbf{n ( \% )}\end{array}$ & $\begin{array}{c}\text { Sexo Masculino } \\
\mathbf{n ( \% )}\end{array}$ & Total $\mathbf{n}(\%)$ \\
\hline AVC no passado & $3(33,3)$ & $6(66,6)$ & $9(15)$ \\
\hline Drogas ilícitas & $1(25)$ & $3(75)$ & $4(6,6)$ \\
\hline Tabagista & $4(26,6)$ & $11(73,3)$ & $15(20)$ \\
\hline Etilista & $1(8,3)$ & $11(91,6)$ & $12(20)$ \\
\hline HAS & $12(44,4)$ & $4(66,6)$ & $16(45)$ \\
\hline Dislipidemia & $2(33,3)$ & $4(77,7)$ & $6(10)$ \\
\hline DM & $5(41,6)$ & $7(58,3)$ & $12(20)$ \\
\hline HIV & $3(30)$ & $7(70)$ & $10(16,7)$ \\
\hline
\end{tabular}

Legenda: AVC (Acidente Vascular Cerebral); DM (Diabetes Mellitus); HAS (Hipertensão Arterial Sistêmica); HIV (Human Immunodeficiency Virus).

Foram constatados nove sujeitos (15\%) que tiveram AVC no passado, sendo que três apresentaram novo episódio de AVC hemorrágico (AVCh) e um de AVC isquêmico (AVCi); três apresentaram outros acometimentos (mielites, lesões medulares e síndromes) e dois lesão neurológica por coinfecção.

Dos seis sujeitos (10\% da amostra) dislipidêmicos, três tiveram AVCh e três AVCi, dois apresentaram tumor de SNC e um outros acometimentos (mielites, lesões medulares, síndromes).

Dos 12 sujeitos ( $20 \%$ da amostra) que têm DM quatro foram acometidos por tumor de SNC, dois por AVCh, dois por mielite, um por AVCi, um por lesão medular, um por síndrome neurológica e um lesão neurológica por coinfecção.

Os quatro (100\%) usuários de drogas ilícitas apresentaram lesão neurológica por HIV.

Dos $12(20 \%)$ sujeitos etilistas, quatro tiveram AVCh, três tumor de SNC, dois lesão neurológica por coinfecção e três outros acometimentos (mielites, lesões medulares, síndromes).

Dos 27(45\%) que apresentaram HAS, quatro tiveram tumor de SNC, um TCE, dois AVCi, 11 AVCh e sete outros acometimentos. Dos que tiveram AVCh a maioria 11 (73,33\%) tinham HAS. 
Dos sujeitos tabagista 15 (25\%), quatro tiveram outros acometimentos, três tumor de SNC, três lesão neurológica por coinfecção, dois AVCh, dois AVCi e um TCE.

Quanto ao município de procedência dos sujeitos desta pesquisa (Tabela 5), obteve-se que a maioria (55,3\%) era do município de Santa Maria; os demais de municípios pequenos e bem pequenos que totalizaram 203.447 habitantes (IBGE,2010). Ressalta-se que o hospital recebe pacientes de 32 municípios.

Tabela 5 - Município de Procedência

\begin{tabular}{c|c|c}
\hline Município de Procedência & $\mathbf{n ( \% )}$ & $\begin{array}{c}\text { Habitantes } \\
(\mathbf{m i l )}\end{array}$ \\
\hline Santa Maria & $33(55,3)$ & 243.544 \\
\hline Formigueiro & $4(6,6)$ & 7.014 \\
\hline São Sepé & $3(5,0)$ & 23.798 \\
\hline São Pedro & $3(5,0)$ & 16.368 \\
\hline São Borja & $3(5,0)$ & 61.671 \\
\hline Dona Francisca & $2(3,3)$ & 3.401 \\
\hline Cacequi & $2(3,3)$ & 13.686 \\
\hline Julio de Castilhos & $2(3,3)$ & 19.579 \\
\hline Paraíso do Sul & $2(3,3)$ & 7.336 \\
\hline São Vicente do Sul & $2(3,3)$ & 8.440 \\
\hline Outros Municípios & & $11.880 ;$ \\
& $4(6,6)$ & $2.952 ;$ \\
& & 11.8473 \\
\hline
\end{tabular}

Legenda: Outros Municípios (Salto do Jacuí, Toropi, Restinga Seca e Jaguari)

Destaca-se que dos sujeitos que sofreram TCE, a maioria - seis (75\%) - era Santa Maria. Também eram da referida cidade, cinco (55\%) sujeitos dos nove (15\% da amostra total da amostra) que tiveram tumor no SNC e cinco $(50 \%)$ dos $10(16,7 \%$ da amostra total) que tiveram lesão neurológica por coinfecção.

A maioria dos sujeitos que tiveram AVCi - quatro (80\%) - era procedente de outros municípios como Dona Francisca, Toropi, São Pedro e Paraíso do Sul.

\section{Discussões}

A maioria dos sujeitos desse estudo é do sexo masculino e da cor branca, resultado que concorda com outro estudo ${ }^{21}$, realizado na mesma região, porém dedicado exclusivamente à população acometida por AVC. A propósito, não se encontrou literatura referente à caracterização de pessoas internadas por LEA, incluindo as diferentes e possíveis etiologias, como o que se faz neste estudo.

Com relação à faixa etária, os resultados deste estudo indicaram uma média de idade ligeiramente mais baixa que a apresentada em estudo realizado em $2013^{22}$, no norte de Portugal. Tal como no caso do sexo, a diferença na faixa etária pode estar relacionada ao fato de que o referido estudo dedicou-se somente à lesão por AVC e este incluiu lesões por TCE e por coinfecções, as quais predominam em faixa mais jovem ${ }^{5,6,23}$.

Quanto ao tipo de LEA, os AVC foram os mais frequentes, tal como em outros estudos ${ }^{21,24}$. No entanto, prevaleceu 0 AVCh, diferentemente de estudos realizados na Região Sul do Brasi ${ }^{21}$ e na Região Norte de Portugal ${ }^{22}$, que apresentaram o AVCi como o mais recorrente. Tal resultado pode ser explicado pelo fato de que esta pesquisa foi realizada em um Hospital de Alta Complexidade, de referência regional no interior do Rio Grande do Sul, o que acaba determinando encaminhamentos de casos mais graves, no caso, o AVCh. Estudo internacional ${ }^{25}$ indicou que, embora o AVCh seja 0 menos frequente, ele corresponde à forma mais grave, pois acarreta maiores complicações neurológicas. Isso porque, os 
AVCh são, geralmente, causados por aneurisma ou traumas extravasculares do cérebro. Os fatores que mais contribuem para um aneurisma são os defeitos de desenvolvimento que causam fraqueza na parede do vaso sanguíneo. Por outro lado, as hemorragias cerebrais também podem ocorrer por Hipertensão Arterial Sistêmica (HAS) ${ }^{26}$.

Neste estudo prevaleceu o acometimento de AVCi no sexo feminino (Tabela 2), conforme outro estudo ${ }^{22}$. A diferença percentual de AVC entre homens e mulheres é relativamente coincidente com uma pesquisa ${ }^{21}$ realizada com o dobro da amostra deste estudo.

Na presente pesquisa (Tabela 3), a HAS associou-se ao DM e à dislipidemia, na faixa etária acima dos 40 anos, concordando com um estudo ${ }^{27}$ o qual destacou que estas três condições de saúde estão entre os principais fatores de risco para doenças cardiovasculares e cerebrovasculares. AHAS foi maior entre as mulheres (Tabela 4). Dado semelhante também foi encontrado em outro estudo ${ }^{28}$ e explicado que, apesar de as mulheres serem as que mais procuram por serviços básicos de saúde, estes não estariam sendo eficientes. Pode-se supor que o mesmo esteja acontecendo nos municípios pesquisados neste estudo.

O mesmo estudo ${ }^{27}$ afirmou que o índice de HAS aumenta na faixa etária dos 60 aos 70 anos, resultados também encontrados neste estudo (Tabela 3). A propósito, outro estudo24 referiu que a incidência de AVC duplica a cada década após os 55 anos, sendo que a maioria dos AVC ocorre após os 65 anos. Também informou que o índice de AVC é influenciado pelos fatores de risco, aumento da expectativa de vida e envelhecimento populacional ${ }^{24}$.

Os resultados deste estudo (Tabelas 3 e 4) espelharam a realidade brasileira das LEA ${ }^{29}$ : altas taxas de HAS, tabagismo, etilismo e DM.

Este estudo revelou fatores de risco para AVC, em ambos os sexos. No feminino, os fatores de risco foram HAS e DM, tal como 0 encontrado na literatura ${ }^{30} \mathrm{e}$, no sexo masculino, os fatores prevalentes foram o tabagismo, o etilismo e a DM, contrariamente a um estudo31 que encontrou a HAS com prevalência semelhante entre homens e mulheres. Ressalta-se que, neste estudo, os fatores de risco estiveram presentes, especialmente, nos casos de AVCh e neoplasia, concordando com as decrições do Plano de Ações Estratégicas para o Enfrentamento das DCNT, no Brasil, 2011-20224.

A recorrência de AVC, um importante resultado deste estudo, encontra ressonância em estudo ${ }^{29}$ que se dedicou à discussão sobre as prioridades para o enfrentamento e a necessidade de investigações acerca das DCNT. A propósito, as DCNT (conforme indicado anteriormente - atualmente chamadas de condições crônicas de saúde) são as principais causas de óbitos no mundo (com um alto número de mortes prematuras), diminuição da qualidade de vida (devido à grande limitação nas atividades de trabalho e de lazer) e criação de impactos econômicos negativos para as famílias, comunidades e sociedades, o que gera maiores iniquidades e aumento da pobreza ${ }^{4}$.

Neste estudo, além da prevalência de diagnóstico de AVCh também foram evidenciados índices de lesão neurológica por coinfecção, distribuída de forma desequilibrada entre os sexos (maior acometimento em homens que em mulheres Tabela 2). Ressalta-se que todos os sujeitos que tiveram lesão neurológica por coinfecção eram portadores de HIV, usuários de drogas ilícitas e com idade entre 18 e 30 anos (Tabela 2). Estudo ${ }^{23}$ realizado em oito cidades brasileiras de grande porte, entre elas Porto Alegre, revelou altas taxas de HIV, bem como alto número de transmissão entre usuários de drogas ilícitas. Estudo recente ${ }^{2}$, de grande magnitude (análise de 1.134 prontuários hospitalares), revelou que 312 sujeitos apresentaram coinfecção (a maioria neurológica) ao HIVIAIDS, sendo que a maioria dos sujeitos era do sexo masculino. As coinfecções são oportunistas e comumente atingem o SNC dos sujeitos com HIV, devido sua condição clínica de imunossupressão ${ }^{23}$. Esse quadro é fortalecido quando associado a fatores de risco como: uso de drogas ilícitas, etilismo, tabagismo, DM e AVC anterior. Note-se que foram encontrados, neste estudo - realizado com populações de municípios de médio e pequeno porte -, dados similares aos encontrados em estudos realizados em cidades de grande porte.

Também houve significativo número de sujeitos do sexo masculino acometidos por tumores de SNC (Tabela 1) com idade superior a 50 anos (Tabela 2). Estudos ${ }^{33,34}$, internacional e nacional - respectivamente -, observam discreto predomínio de tumores de SNC no sexo masculino. Estima-se que os tumores do SNC são a terceira causa de mortes entre 15 e 34 anos de idade e a quarta em homens entre 35 e 54 anos $^{34}$.

Este estudo revelou que o consumo de bebidas alcoólicas é um fator de risco em todas as faixas etárias (Tabela

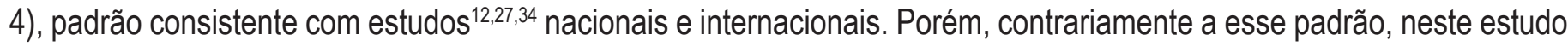
tal fator não está relacionado aos acidentes de viação e acometimentos por TCE. Tal achado, possivelmente, está relacionado ao curto período de coleta e em mês sem feriados prolongados, sabidamente circunstâncias favorecedoras de acidentes e ferimentos por violência. 
Os casos de TCE prevaleceram no sexo masculino (Tabela 1) e na idade de 18 a 50 anos (Tabela 2) corroborando com estudo 6 realizado na Região Nordeste do Brasil, em 2012.

Quanto à procedência, este estudo revelou que a maioria dos sujeitos que sofreu AVCh, TCE, neoplasia e lesão neurológica por HIV advém do município de Santa Maria, enquanto que a maioria dos que tiveram AVCi é procedente de municípios pequenos (Tabela 5). A primeira situação pode ser explicada pelo fato de existir, no referido município, outros hospitais em condições de realizar tratamento de casos mais simples, encaminhando para o hospital de alta complexidade os casos que exigem recursos tecnológicos mais sofisticados. Enquanto que na segunda situação, inexistem nos pequenos municípios serviços hospitalares que possam realizar cuidados emergenciais e/ou urgentes. A esse respeito, não foram encontrados estudos similares; entretanto, é possível discutir que o perfil de morbidade e mortalidade de uma determinada população está relacionado às características regionais de onde ela se encontra.

Sabe-se que as condições socioeconômicas do município de Santa Maria e região estão ligadas fortemente ligadas ao comércio e à agropecuária familiar, tendo, portanto, restritos recursos locais e regionais, de modo que os investimentos na área da saúde dependem do estado e da federação e, consequentemente, o acesso aos serviços secundários e terciários de saúde é fortemente dependente de serviços como o Hospital Universitário, onde foi realizada esta pesquisa.

O município de Santa Maria e os da região detêm gestão plena na Atenção Básica, nível de atenção à saúde onde podem ser bem abordados os fatores de risco que produzem LEA; para tanto é necessária educação permanente dos trabalhadores da Atenção Básica e educação popular visando modificar os hábitos deletérios que potencializam a ocorrência de LEA, no município e da região.

\section{Considerações Finais}

Os dados deste estudo, apesar de parciais, revelam que o Hospital Universitário Regional atende as populações do município sede do Hospital, as dos municípios de sua abrangência, bem como as de regiões mais distantes.

Os casos prevalentes foram adultos do sexo masculino e da cor branca; sendo alto o índice de AVCh, seguido de lesão neurológica por coinfecção e Tumor de Sistema Nervoso Central. Estes associados aos fatores de risco: DM, HAS, etilismo, tabagismo, HIV e uso de drogas ilícitas.

A maioria dos fatores de risco encontrados é decorrente de causas evitáveis, o que pode ser interpretado como insuficiência e/ou ineficiência das ações preventivas relacionadas às condições crônicas de saúde (doenças vasculares e neoplásicas, por exemplo) e às infectocontagiosas que afetam o SNC, o que, por sua vez, requer atenção dos trabalhadores de saúde dos diferentes níveis de atenção à saúde.

Estudos como este, com período de coleta curto, ou com períodos dilatados, são de extrema importância, pois, além de serem escassos, conseguem identificar a incidência de patologias e perfis de sujeitos de uma região, favorecendo uma reflexão e/ou verificação de políticas voltadas para a promoção, proteção e recuperação da saúde, ou seja, estudos como este possibilitam análises e reelaboração das práticas educacionais em saúde, de acordo com as necessidades das populações de uma dada região.

\section{Referências}

1. Malta DC, Silva JRJB. O Plano de Ações Estratégicas para o Enfrentamento das Doenças Crônicas Não Transmissíveis no Brasil e a definição das metas globais para o enfrentamento dessas doenças até 2025: uma revisão. Epidemiologia e Serviços de Saúde. 2013; 22(1):151-164.

2. Reis Ferreira TC, Souza APC, Júnior RSR. Perfil clínico e epidemiológico dos portadores do HIVIAids com coinfecção de uma unidade de referência especializada em doenças infecciosas parasitárias. Revista da Universidade Vale do Rio Verde 2015; 13(1): 419-431.

3. Soares MT et al. Prevalência das coinfecções em pacientes notificados com Aids no Centro de Referência na Paraíba. Revista Brasileira de Ciências da Saúde 2014;18: 5-12.

4. Brasil. Ministério da Saúde, Secretaria de Vigilância em Saúde, Departamento de Análise de Situação de Saúde. Plano de ações estratégicas para o enfrentamento das doenças crônicas não transmissíveis (DCNT) no Brasil 2011-2022 / Ministério da Saúde, Secretaria de Vigilância em Saúde, Departamento de Análise de Situação de Saúde. - Brasília: Ministério da Saúde; 2011 [acesso em 11 outubro 2015]. Disponível em: http://189.28.128.100/dab/docs/geral/plano_dcnt.pdf. 
5. Santos M E. Traumatismos Crânio-Encefálicos: características e evolução. Psicologia 2014;16 (1): 97-122.

6. Dantas IEF, Oliveira TT, Neto CDM. Epidemiologia do traumatismo crânio encefálico (TCE) no Nordeste no ano de 2012. Revista Brasileira de Educação e Saúde 2014; 4(1): 18-23.

7. Brain Injury Suport. Supporting New Zealandres Living With Brain Injury. Nova Zalândia. Disponível em: http://www. brain-injury.org.nz/html/what_is_brain_injury_html ( acesso 08 de nov de 2015).

8. Alonso GSO, Silveira VC. Lesões encefálicas adquiridas. In: Fernandes AC, Ramos ACR, Casalis MEP, Hebert SK. Medicina e reabilitação: princípios e prática. São Paulo: Artes Médicas; 2007. p.161.

9. Brasil, Ministério da Saúde. Indicadores e dados básicos - Brasil 2011. Indicadores de mortalidade. DATASUS Informações de Saúde - TABNET. Indicadores de saúde; 2010 [acesso em 2015 outubro 31]. Disponível em: http://tabnet. datasus.gov.br/cgi/deftohtm.exe?idb2011/c08.def.

10. Brasil. Ministério da Saúde, Secretaria de Atenção à Saúde, Departamento de Atenção Especializada. Manual de rotinas para atenção ao AVC / Ministério da Saúde, Secretaria de Atenção à Saúde, Departamento de Atenção Especializada. - Brasília: Editora do Ministério da Saúde; 2013 [acesso em 2015 novembro 04]. Disponível em: http:/l bvsms.saude.gov.br/bvs/publicacoes/manual_rotinas_para_atencao_avc.pdf.

11. Schmidt $\mathrm{Ml}$ et al. Chronic non communicable diseases in Brazil: burden and current challenges. Lancet. 2011;377(9781):1949-61.

12. World Health Organization. 2008-2013 Action plan for the global strategy for the prevention and control of non communicable disease [Internet]. Geneva: World Health Organization; 2008 [cited 2013 Sep 12]. Available from: http:// whqlibdoc.who.int/publications/2009/9789241597418_eng.pdf

13. Alwan A et al. Monitoring and surveillance of chronic non-communicable diseases: progress and capacity in highburden countries. Lancet. 2010; 376(9755):1861-68.

14. Gentile JKA et al. Condutas no paciente com trauma cranioencefálico: [revisão]. Rev. Soc. Bras. Clín. Méd 2011;9(1): 74-82.

15. Hoffman S, Propp JM, Mccarthy BJ. Temporal trends in incidence of primary brain tumors in the United States, 19851999. Neuro Oncol. 2006;8(1):27-37.

16. Monteiro GTR, Koifman S. Mortalidade por tumores de cérebro no Brasil, 1980-1998. Cad Saúde Públ. 2003;19(4):1139-51. 17. El-zein R, Minn AY, Wrensch M, Bondy ML. Epidemiology of brain tumors. Cancer in the nervous system. New York: Oxford University Press; 2002 p. 252-66.

18. Medeiros CAM et al. Perímetro cervical, uma medida à beira do leito relacionada com a mortalidade no acidente vascular cerebral isquêmico. Rev Assoc Med Bras 2011;57(5): 559-64.

19. Pereira CU, Jesus RM. Epidemiology of spinal injury in Aracaju. A prospective series. J Bras Neurocirurg 2011;22(2):26-31.

20. Chen Y, Tang Y, Vogel LC, Devivo MJ. Causes of spinal cord injury. Top Spinal Cord Inj Rehabil 2013;19(1):1-8.

21. Sá BP, Grave MTQ, Périco E. Profile of patients hospitali-zed with Stroke in a hospital of Vale do Taquari. Epilepsia 2014;3(2): 4.

22. Moutinho M, et al. Avaliação da via verde do acidente vascular cerebral no norte de Portugal. Acta Med Port 2013;26(2):113-122.

23. Martins T et al. Cenário Epidemiológico da Infecção pelo HIV e AIDS no Mundo. Fisioterapia \& Saúde Funcional 2014;3(1): 4-7.

24. Piassaroli CAP, Almeida GC, Luvizotto JC, Suzan ABBM. Modelos de reabilitação fisioterápica em pacientes adultos com sequelas de AVC isquêmico. Rev Neurocien 2012; 20:128-37.

25. Schepers VP, Ketelaar M, Visser-Meily AJ, de Groot V, Twisk JW, Lindeman E. Functional recovery differs between ischaemic and haemorrhagic stroke patients. J Rehabil Med. 2008; 40(6):487-489.

26. Sullivan SB, Schmitz TJ. Fisioterapia avaliação e tratamento. $4^{a}$ Ed. Barueri: Manole, 2004, p.540.

27. Malta DC, Oliveira MR, Moura EC, Silva AS, Zouain CS, Santos FP, et al. Fatores de risco e proteção para doenças crônicas não transmissíveis entre beneficiários da saúde suplementar: resultados do inquérito telefônico Vigitel, Brasil, 2008. Ciênc Saúde Coletiva. 2011; 16(3):2011-22.

28. Malta DC, Sarnovi F. Freqüência de hipertensão arterial e fatores associados: Brasil, 2006. Rev Saúde Pública. 2009;43(n. Supl 2):98-106.

29. Duncan BB. et al. Doenças crônicas não transmissíveis no Brasil: prioridade para enfrentamento e investigação. Rev Saúde Pública. 2012; 46(1): 126-134. 
30. Costa JSD et al. Prevalência de hipertensão arterial em adultos e fatores associados: um estudo de bases populacional urbana em Pelotas Rio Grande do Sul, Brasil. Rev Bras Cardiol 2007; 88:59-65. http://dx.doi.org/10.1590/ S0066-782X2007000100010

31. Brandão AA et al. Conceituação, epidemiológica e prevenção primária. J Bras Nefrol 2010;32 (supl 1):1-4. http:// dx.doi.org/10.1590/S0101-28002010000500003

32. Oliveira RM. C. Afecções neurológicas do sistema nervoso central. In:Levy J,Oliveira ASB. Reabilitação em doenças neurológicas: guia terapêutico prático. São Paulo:Atheneu;2003.p.15-301.

33. Raaschou ON et al. Increasing incidence of childhood tumours of the central nervous system in Denmark, 1980-1996. Br J Cancer. 2006;95(3):416-22.

34. Mascarenhas MDM et al. Atendimentos de emergência por causas externas e consumo de bebida alcoólica - Capitais e Distrito Federal, Brasil, 2011. Ciênc. Saúde Coletiva. 2015; 20(4): 1037-1046.

\section{Jodéli Pommerehn}

Endereço para correspondência - Rua: Senador Correa, $n^{\circ} 439$,

Bairro: Centro, CEP: 96200-600, Rio Grande, RS, Brasil.

E-mail: jodelipo@gmail.com

Lattes: http://lattes.cnpq.br/6829352998322404

Elenir Fedoose - efedosse@gmail.com

\section{Enviado em 23 de janeiro de 2017. Aceito em 11 de agosto de 2017.}

